Ökologie \& Landbau, der AGÖL oder von Umweltverbänden ein Anteil von 10-20 Prozent Ökolandbau bis spätestens 2010 angestrebt.

Welche Rolle künftig der Regionalvermarktung zukommt, ist kaum absehbar. Trotz zahl- und erfolgreicher Modellprojekte gibt es innerhalb des Ökolandbaus widerstrebende Tendenzen. Einerseits gilt es sich dem konventionellen Handel zu öffnen - mit der Gefahr entsprechender Rationalisierung und Zentralisierung. Andererseits mehren sich die Stimmen, die den damit drohenden Verlust an Nachhaltigkeit anprangern und eine stärkere Rückbesinnung auf die Region einfordern. Vieles wird von der Initiative auf kommunaler und regionaler Ebene, aber auch von der Gestaltung der politischen Rahmenbedingungen wie der Entwicklung der ökologischen Steuerreform oder Förderprogrammen für Regionalvermarktung abhängen.

Trotz heftiger Diskussionen - auch durch das „Zukunftsfähige Deutschland“ - hat sich auf dem Weg zu einer nachhaltigen Land- und Ernährungswirtschaft nicht viel getan. Die zunehmenden Skandale in Teilen der konventionellen
Landwirtschaft und der Nahrungsmittelindustrie eröffnen allerdings wachsende Chancen für die kontrollierte ökologische Landwirtschaft mit einer klar definierten und rechtlich abgesicherten Qualitätssicherung sowie für die Regionalisierung mit einer gesicherten und transparenten Herkunft. Die vielen lokalen und regionalen Initiativen weisen damit den Weg hin zu einem breiten gesellschaftlichen Trend.

\section{Anmerkungen}

(1) BUND/Misereor (Hrsg.): Zukunftsfähiges Deutschland. Basel 1996.

(2) Rosenberger, M.: Die Studie „Zukunftsfähiges Deutschland” in der Fachdiskussion. In: Hermle, R. (Hrsg.):

Ein Buch macht von sich reden. Misereor, Aachen 1997, S. $61-95$.

(3) Sonnleitner, G.: Rundbrief an die Kreisbäuerinnen und Kreisobmänner des Bayerischen Bavernverbandes.

2.4.1996.

(4) Stoiber, E.: Manuskript der Rede des Bayerischen Ministerpräsidenten anläßlich der Eröffnung der Misereor-Fastenaktion in Eichstätt. 16.2.1997.

(5) DBV: Nachhaltigkeit ist keine Worthülse. DBV-Informationen vom 20.5.1999, S. 7-9.
(6) Stiffung Ökologie \& Landbau (Hrsg.): Ökologie \& Landbau, Heft 114 (2000), S. 2.

(7) Stiffung Ökologie \& Landbau (Hrsg.): Ökologie \& Landbau, Heft 112 (1999), S. 2.

(8) 0.V.: Funke hält nichts von Cross compliance und Modulation. In: Agra-Europe, Heft 22/1999, S.14.

(9) Jasper, U.: Agenda 2000 - es kam anders als erhofft. In: Agrarbündnis (Hrsg.): Kritischer Agrarbericht 2000, S. 37-41.

(10) Umweltbundesamt (Hrsg.): Nachhaltiges Deutschland. Berlin 1997, S. $163 \mathrm{ff}$.

\section{Der Autor}

Bernhard Burdick ist Projektleiter für Nachhaltige Regionalentwicklung, Land- und Ernährungswirtschaft am Wuppertal Institut.

Kontakt: Wuppertal Institut für Klima, Umwelt, Energie, Döppersberg 19, 42103 Wuppertal,

Tel. 0202/ 2492-110, Fax -250

\title{
Landwirtschaft contra Naturschutz?
}

\section{Nachhaltige Landwirtschaft ist ohne den Schutz der natürlichen Ressourcen als eigene Produktionsgrundlage nicht denkbar. Aber auch ein nachhaltiger und flä- chendeckender Naturschutz ist in der fast durchgängig genutzten Kulturland- schaft Deutschlands ohne die Einbeziehung der Landwirtschaft nicht erreichbar. Dieses besondere Verhältnis von Landwirtschaft und Naturschutz wird am der- zeit aktuellen Beispiel der Eingriffsregelung beleuchtet.}

$\mathrm{D}$

Von Jens-Peter Abresch Naturschutzpolitik. Das Naturschutzrecht (Bundesnaturschutzgesetz und Ländernaturschutzgesetze) und das Baurecht enthalten die rechtlichen Grundlagen. Das Prinzip der Eingriffsregelung ist leicht einsichtig und lautet vereinfacht: Wer natürliche Ressourcen verbraucht, der ist zur Schadenskompensation verpflichtet. Die Naturschutzpläne entwickeln dazu Kompensationsmaßnahmen, die den jeweiligen Biotopwert- und Bilanzierungskonventionen genügen. Nachdem jahrelang vor allem Bilanzierungsmethoden und Bewertungsfragen disku- tiert wurden, rückt zunehmend die Problematik des Vollzuges und der Dauerhaftigkeit der Kompensationseffekte der Eingriffsregelung in den Vordergrund. In den wenigen vorliegenden Erfolgsbetrachtungen zur Eingriffsregelung zeichnet sich dabei ein bedenkliches Bild ab (1).

Zum Teil werden nur geringe Anteile geplanter Kompensationsmaßnahmen in der Praxis umgesetzt. Besonders die Pflege und Entwicklung von Ersatzflächen ist häufig nicht gesichert. Die landschaftspflegerischen Begleitplanungen erweisen sich mit Blick auf die Verfügbarkeit der beplanten Flächen oftmals als schwer umsetzbar. Im Unterschied zum Plan stehen im Ergebnis dann häufig Flickenteppiche von Kompensationsmaßnahmen mit teilweise zweifelhaftem Bezug zu den Eingriffseffekten und vernachlässigbaren landschaftsökologischen Effekten im betroffenen Landschaftsraum. Auch Fragen der Kosteneffizienz werden bislang kaum thematisiert. Unabhängig davon wie kritisch man die Erfolge der Eingriffsregelung im Einzelnen beurteilt: Unstrittig ist, dass Umsetzungsprobleme bestehen und die Wirksamkeit des Instrumentes hinter den Erwartungen zurïckbleibt. Beleg für diese Situation ist auch die breite Diskussion zur Weiterentwicklung und Flexibilisierung der Eingriffsregelung (2).

\section{Landwirtschaft und Eingriffsregelung}

Landwirtschaftliche Betriebe erleben die Eingriffsregelung oft als problematisches Instrument. Zunächst sehen sich die Landwirte den Flächenverlust durch das Eingriffsvorhaben gegenüber Dabei verkehrt sich das Problem der Flächenverluste häufig zur Chance, Land zu einem attraktiven Veräußerungspreis zu verkaufen. Wenn aber die Flächenansprüche zur Kompensation an die Landwirte herangetragen werden, spitzen sich die Konflikte zu. Die Flächenentzüge werfen zum Teil erhebliche agrarstrukturelle und einzelbetriebliche Probleme auf, beispielsweise die Pachtflächenproblematik: Landnutzer und Flächeneigen- 
tümer fallen zunehmend auseinander. Bei Veräußerung von Flächen für Kompensationszwecke geraten deshalb die pachtenden Betriebe leicht in kritische Situationen. Nutzfläche ist nicht vermehrbar und alternative Flächen am Bodenmarkt sind nicht beliebig verfügbar. Gerade in Realteilungsgebieten können Flächenveräußerungen durch außerlandwirtschaftliche Eigentümer zu Verwerfungen im fragilen System von gegenseitiger Verpachtung und Unterverpachtung führen. Damit wird der teilweise rigide Widerstand der Landwirtschaft bei Eingriffskompensationen aus betrieblicher Sicht nachvollziehbar. Solange die Kompensationsmaßnahmen nicht in Abstimmung und Kooperation mit den Landnutzern entwickelt werden, wird die Eingriffsregelung in vielen Fällen ihre Probleme kaum überwinden.

\section{- Vertragsnaturschutz als Lösung}

Der hier vorgestellte Lösungsansatz zielt auf eine Flexibilisierung von Kompensationstatbeständen im Rahmen der Eingriffsregelung ab. Neben dem Erwerb von Flächen und der Realisierung investiver Maßnahmen, die das Problem der dauerhaften Pflege nach sich ziehen, kann eine definierte, naturschutzorientierte Bewirtschaftung landwirtschaftlicher Flächen als Kompensation von Eingriffen ausgestaltet werden. Für die kommunale Bauleitplanung werden zum Beispiel folgende Bausteine vorgeschlagen:

Vertragsnaturschutz: Im Zuge eines Bebauungsplanes kommt es in einer Stadt zur Erhebung von Kompensationskosten bei den Bauträgern. Im Rahmen der landschaftspflegerischen Begleitplanung werden die Wertverluste im Sinne der Eingriffsregelung naturschutzfachlich analysiert und geeignete Maßnahmen zur Kompensation eingegrenzt. Die Stadt hat damit zwei wesentliche Informationen: Die Geldmenge, die zur Realisierung von Eingriffen zur Verfügung steht und die naturschutzfachliche Formulierung der Kompensationsmaßnahmen.

Nach dem vorgeschlagenen Modell kann die Stadt ausgesuchte Maßnahmen, die als Landschaftspflegeleistungen darstellbar sind, öffentlich ausschreiben. Die landwirtschaftlichen Betriebe können mit ihren Flächen Angebote zur Leistungserstellung einbringen. Der Landwirt bietet also Betriebsflächen an, um darauf die nachgefragten Landschaftspflegeleistungen selbst zu realisieren. Der Preis für die Leistungen bildet sich dann in den Vertragsverhandlungen zwischen Landwirt und Stadt (3). Finden sich anbietende landwirtschaftliche Betriebe, so kann ein
Vertrag abgeschlossen werden, ähnlich den bereits praktizierten Vertragsnaturschutzmodellen. Die Vertragsdauer sollte zwischen 25 und 30 Jahren liegen. Der Landwirt verpflichtet sich für den Vertragszeitraum zur Sicherstellung der definierten Pflegeleistung auf seiner Fläche.

Für das Vertragsnaturschutzmodell mit langen Laufzeiten bieten sich Finanzierungsmodalitäten außerhalb der kommunalen Haushalte an. Vorgeschlagen wird ein Fondsmodell. Gelder, die von den Ausgleichsverpflichteten an die Städte/ Gemeinden geflossen sind, werden in den Fond abgeführt. Das Fondsvermögen wird zweckgebunden und zinsbringend angelegt. Die Verträge mit den Landwirten sollen dann langfristig unter Erhalt des Kapitalstockes aus dem Fond finanziert werden. Zur Eingriffskompensationen durch Landschaftspflegeleistungen werden nur die Zinserträge herangezogen. In ersten Modellrechnungen konnte gezeigt werden, dass bei einer Finanzierung von Landschaftspflegeleistungen mit der Landwirtschaft und unter Verwendung gültiger Biotopwertkonventionen alleine unter Verwendung der Zinserträge ein Wertausgleich im Sinne der Eingriffsregelung realisierbar ist (4). Der Wertverlust in Landschaften durch Eingriffe wird monetarisiert und ermöglicht so langfristig landschaftsökologisch wirksame Kompensationsmaßnahmen.

\section{- Ausblick}

Mit Blick auf die skizzierten Probleme der Eingriffsregelung und ihre landwirtschaftlichen Implikationen liegen die Vorteile des Ansatzes auf der Hand. Die Flächenentzüge durch Kompensationsmaßnahmen werden eingedämmt. Die Landwirtschaft in den betroffenen Regionen wird vom Flächenbereitsteller zu einem aktiven Partner in der Kompensation von Eingriffen. Auch die Pflegeproblematik wird entschärft. Letztlich eröffnet ein solches Vorgehen den landwirtschaftlichen Betrieben eine leistungsbezogene Honorierung ökologischer Leistungen auf eigenen Flächen, also auch Einkommensbeiträge, die zur agrarstrukturellen Stabilisierung besonders an marginalen Produktionsstandorten führt. Erste praktische Erprobungen zeigen, dass der Ansatz grundsätzlich tragfähig und umsetzbar ist (5). Gleichwohl ist der Ansatz mit einer Reihe ungelöster Probleme verbunden. Deshalb fördert die Deutsche Bundesstiftung Umwelt ein Forschungsvorhaben, bei dem der skizzierte Ansatz in zwei Beispielsregionen erprobt und weiter entwickelt wird (6). Bereits heute kann aber festgehalten werden: Die Eingriffsregelung nach dem Raumordnungsgesetz und die Praxis der naturschutzrechtlichen Eingriffsregelung enthalten Handlungsspielräume für eine stärkere Einbeziehung von Landschaftspflegeleistungen der Landwirtschaft. Es kommt praktisch vor allem darauf an, dass Landwirtschaft und Naturschutz gemeinsam und ohne tradierte Vorbehalte nach Lösungen mit gegenseitigen Nutzeneffekten suchen. Gelingt dies, kann mit Berechtigung von einem praktischen Beispiel für eine nachhaltige Verknüpfung von Naturschutz und Landwirtschaft gesprochen werden.

\section{Anmerkungen}

(1) Schwoon, G.: Sicherung, Pflege und Kontrolle von Kompensationsmaßnahmen am Beispiel von Straßenbauvorhaben des Bundes und des Landes Niedersachsen. Diplomarbeit, Universität Hannover 1996.

(2) Jessel, B: Wie zukunftsfähig ist die Eingriffsregelung?

In: Naturschutz und Landschaftsplanung, 30 (1998), 7, S. 219-222.

(3) Zur Organisation solcher regionaler Landschaftspflegemärkte sind verschiedene Modelle denkbar und zum Teil bereits in der Erprobung, z.B. Flächenagenturen. Siehe Strasser, H.: Flächen- und Maßnahmenbevorratung im Rahmen der Eingriffsregelung - Flächenpooling - In: ARSU Positionen, Heft 8, S. 36-44, Oldenburg 1999.

(4) Abresch, J.P./ Bauer, S./ Hummelsheim: Gesellschaftliche Funktionen der Landwirtschaft im ländlichen Raum: Eine empirische Untersuchung am Beispiel der hessischen Gemeinden Flörsheim und Lohra, In: Landwirtschaftliche Rentenbank (Hrsg.): Landwirtschaft im ländlichen Raum Funktionen, Formen, Potentiale, Schriftenreihe der Landwirtschaftlichen Rentenbank, Band 11, 1997, S. 93-139. (5) Mit der Stadt Herford und der Landwirtschaftskammer Westfalen hat die Professur für Projekt- und Regionalplanung eine erste praktische Erprobung erreicht. Siehe Asbrand: So macht Naturschutz Spaß. In: Landwirtschaftliches Wochenblatt Westfalen, Nr. 46/1999, S. $18 \mathrm{ff}$.

(6) Abresch, J.P./ Strasser, H.: Landwirtschaft und Eingriffsregelung. Weiterentwicklung des naturschutzrechtlichen Planungsinstruments durch flexible Modelle zur Honorierung kompensationswirksamer Naturschutzleistungen durch die Landwirtschaft. DBU Deutsche Bundesstiftung Umwelt 1999, Projekt-Az. 15813.

\section{Der Autor}

Dr. Jens-Peter Abresch ist wissenschaftlicher Mitarbeiter an der Justus-Liebig-Universität Gießen. Konfakt: JLU Gießen, Professur für Projekt- und Regionalplanung, Diezstraße 15, 35390 Gießen, Tel. 0641/ 99373-16, Fax -19, E-mail: Jens-Peter.Abresch@agrar.uni-giessen.de 
(c) 20I0 Authors; licensee IÖW and oekom verlag. This is an article distributed under the terms of the Creative Commons Attribution Non-Commercial No Derivates License (http://creativecommons.org/licenses/by-nc-nd/3.o/), which permits unrestricted use, distribution, and reproduction in any medium, provided the original work is properly cited. 